\title{
Three-dimensional photoacoustic imaging and inversion for accurate quantification of chromophore distributions
}

\author{
Martina Fonseca, Emma Malone, Felix Lucka*, Rob Ellwood, Lu An, Simon Arridge*, Paul \\ Beard, Ben Cox
Department of Medical Physics and Biomedical Engineering, University College London, Gower Street, London WC1E 6BT, UK; 6BT, UK; \\ *Department of Computer Science, University College London, Gower Street, London WC1E
}

\begin{abstract}
Photoacoustic tomography can, in principle, provide quantitatively accurate, high-resolution, images of chromophore distributions in 3D in vivo. However, achieving this goal requires not only dealing with the optical fluence-related spatial and spectral distortion but also having access to high quality, calibrated, measurements and using image reconstruction algorithms free from inaccurate assumptions. Furthermore, accurate knowledge of experimental parameters, such as the positions of the ultrasound detectors and the illumination pattern, is necessary for the reconstruction step. A meticulous and rigorous experimental phantom study was conducted to show that highly-resolved 3D estimation of chromophore distributions can be achieved: a crucial step towards in vivo implementation. The phantom consisted of four $580 \mu \mathrm{m}$ diameter tubes with different ratios of copper sulphate and nickel sulphate as hemoglobin analogues, submersed in a background medium of intralipid and india ink. The optical absorption, scattering, photostability, and Grüneisen parameter were characterised for all components independently. A V-shaped imaging scanner enabled 3D imaging with the high resolution, high sensitivity, and wide bandwidth characteristic of Fabry-Pérot ultrasound sensors, but without the limited-view disadvantage of single-plane scanners. The optical beam profile and position were determined experimentally. Nine wavelengths between 750 and $1110 \mathrm{~nm}$ were used. The images of the chromophore concentrations were obtained using a model-based, two-step, procedure, that did not require image segmentation. First, the acoustic reconstruction was solved with an iterative time-reversal algorithm to obtain images of the initial acoustic pressure at each of the nine wavelengths for an $18 \times 17 \times 13 \mathrm{~mm}^{3}$ volume with $50 \mu \mathrm{m}$ voxels. Then, 3D high resolution estimates of the chromophore concentrations were obtained by using a diffusion model of light transport in an iterative nonlinear optimisation scheme. Among the lessons to be drawn from this study, one is fundamental: in order to obtain accurate estimates of chromophores (or their ratios) it is not only necessary to model the light fluence accurately, but it is just as crucial to obtain accurate estimates of the initial acoustic pressure distributions, and to account for variations in the thermoelastic efficiency (Grüneisen parameter).
\end{abstract}

Keywords: quantitative photoacoustic tomography, chromophore quantification, model-based inversion, phantom experiment

\section{INTRODUCTION}

The potential to obtain high resolution 3D images of estimates of chromophore concentrations is a highly desirable feature of Photoacoustic Tomography (PAT). The range of chromophores of interest that could be probed include genetically encoded reporters, endogenous chromophores (e.g oxy and deoxy-hemoglobin, lipid or melanin) and exogenous chromophores (e.g. dyes and nanoparticles with the potential of being funcionalised to target a specific process and therefore act as a biomarker). ${ }^{1}$ The ability to map and characterise a wealth of biological processes at the genetic, molecular and cellular level is tantalisingly close. Despite this, only very few studies have rigorously and meticulously explored the degree of accuracy that can be obtained in PAT. ${ }^{2-4}$ These used models of light transport within model-based minimisations to correct for spectral and spatial distortion caused

Send correspondence to Martina Fonseca: E-mail: martina.fonseca.13@ucl.ac.uk

Photons Plus Ultrasound: Imaging and Sensing 2017, edited by Alexander A. Oraevsky, Lihong V. Wang, Proc. of SPIE Vol. 10064, 1006415 · @ 2017 SPIE · CCC code: 1605-7422/17/\$18 · doi: 10.1117/12.2250964 
by the light fluence. Nevertheless, given the high-dimensionality and ill-posedness of the general problem, they were typically applied on 2D slices or were segmented into regions which were assumed to have constant optical properties. While this reduces the number of unknowns considerably, leading to a lower computational load and increased robustness, it imposes strong a-priori assumptions on the underlying chromophore concentrations and therefore significantly reduces the level of spatial detail that is obtainable. In this study, the feasibility of achieving fully-resolved (voxel-by-voxel), volumetric chromophore distribution estimation was explored using a highly controlled phantom experiment. This was divided into the following tasks:

1. Phantom Development - develop a stable and realistic phantom, and accurately characterise its optical, acoustic and thermoelastic properties,

2. Experimental Measurements - make accurate measurements of the acoustic field in absolute units (using a V-shaped array consisting of low-directionality detection elements with sufficiently broad bandwidth), as well as measurements of the experimental setup, including the array geometry, surface illumination, and pulse-to-pulse variation in the laser energy,

3. Acoustic Reconstruction - make a faithful reconstruction of the initial pressure distribution in absolute units from the measured data, minimising the artefacts and incorporating physical prior constraints (nonnegative initial acoustic pressure and zero initial acoustic particle velocity),

4. Optical Reconstruction - estimate chromophore concentrations voxelwise, incorporating prior knowledge (such as the scattering coefficient and Grüneisen parameter).

\section{PHANTOM}

Copper sulphate $\left(\mathrm{CuSO}_{4} .5 \mathrm{H}_{2} \mathrm{O}\right)$ and nickel sulphate $\left(\mathrm{NiSO}_{4} .6 \mathrm{H}_{2} \mathrm{O}\right)$ were chosen as chromophores for their photostability (no photobleaching or ground-state depletion), absorption linearity with concentration, and dissimilar absorption spectra. They have the additional advantage that their spectra make them suitable as surrogates for oxy- and deoxyhemoglobin. The phantom used for this experiment comprised 4 polythene tubes of 580 $\mu \mathrm{m}$ inner diameter and $190 \mu \mathrm{m}$ wall thickness in a background of $1 \% \mathrm{w} / \mathrm{v}$ intralipid and india ink (such that $\left.\mu_{a, \text { indiaink }}(750 \mathrm{~nm}) \simeq 0.0064 \mathrm{~mm}^{-1}\right)$. The tubes were filled with mixtures of $0.5 \mathrm{M}$ copper sulphate mother solution and 2.2 M nickel sulphate mother solution, such that $Q=\{25,100,75,0\} \%$ from top to bottom tube respectively (Figure 1 (a) ), where the ratiometric quantity

$$
Q(\%)=\frac{\frac{c_{N i S O_{4}}}{2.2}}{\frac{c_{C u S O_{4}}}{0.5}+\frac{c_{N i S O_{4}}}{2.2}} \times 100
$$

is introduced as an analogue for oxygen saturation, $\mathrm{SO}_{2}$. The resulting absorption coefficient spectra of the four tubes are shown in Figure 1 (b), as measured by a dual-beam spectrophotometer (Lambda 750, Perkin Elmer, Waltham, MA, USA). The absorption of the background is also displayed, having been computed as a joint contribution of water absorption ${ }^{5}$ and india ink (mother solution measured in spectrophotometer). Figure 1 (c) shows the measured reduced scattering characteristics of the background medium obtained through total transmittance and reflectance measurements in a $100 \mathrm{~mm}$ integrating sphere mounted in the spectrophotometer, followed by application of the inverse adding-doubling method. ${ }^{6}$ The Grüneisen parameter of the sulphate compounds was found, using photoacoustic spectroscopy ${ }^{7}$ to have a linear dependence with concentration such that for a given aqueous solution:

$$
\Gamma_{\text {solution }}=\Gamma_{\text {water }}\left(1+\beta_{\mathrm{NiSO}_{4}} c_{\mathrm{NiSO}_{4}}+\beta_{\mathrm{CuSO}_{4}} c_{\mathrm{CuSO}_{4}}\right)
$$

where $\Gamma_{\text {water }}$ is the Grüneisen parameter of water and parameters $\beta_{\mathrm{NiSO}_{4}}=0.3247 M^{-1}$ and $\beta_{\mathrm{CuSO}}=$ $0.7078 M^{-1}$ give the slope of the linear dependency for each compound. Finally, the speed of sound in the medium, $c_{s}$, was set to literature values of water at bath temperature, ${ }^{8}$ since intralipid concentration of $1 \% \mathrm{w} / \mathrm{v}$ has been shown to have negligible effect on the speed of sound in water. ${ }^{9}$ 


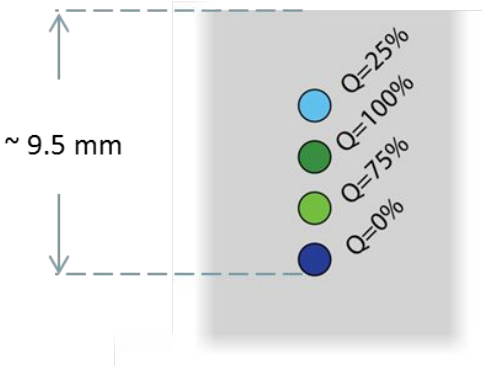

(a) Tube arrangement schematic

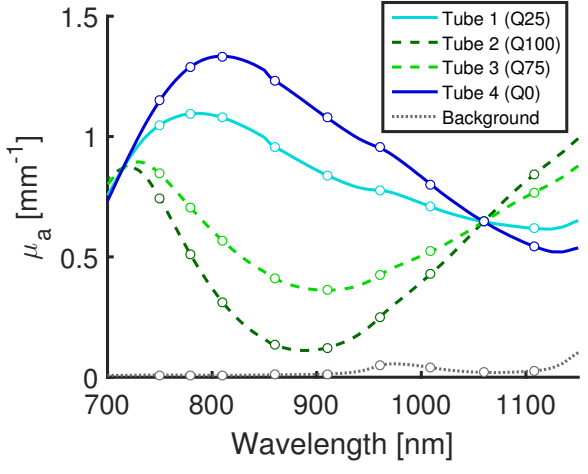

(b) Optical absorption

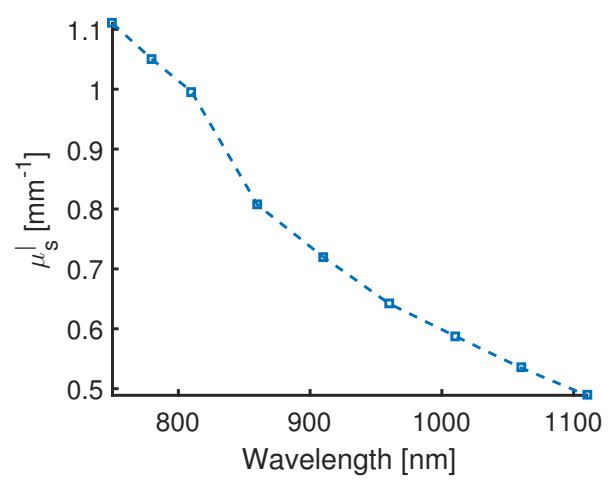

(c) Background reduced scattering

Figure 1: (a) Schematic of the tubes, filled with mixtures of $\mathrm{CuSO}_{4}$ and $\mathrm{NiSO}_{4}$, in a background of intralipid and india ink. (b) Optical absorption spectra of each of the four tubes and of the background. (c) Reduced scattering of the background medium.

\section{EXPERIMENTAL MEASUREMENTS}

Fabry-Pérot sensor arrays were used to measure the photoacoustic (PA) signals, as they provide wide bandwidth (DC to $\sim 40 \mathrm{MHz}$ ), low noise-equivalent pressure (<200 Pa peak amplitude) and near omni-directionality. ${ }^{10}$ Here, two Fabry-Pérot sensors were employed in an orthogonal arrangement, ${ }^{11,12}$ to reduce limited-view effects ${ }^{13,14}$ (Figure 2). To obtain PA signals in units of Pascals, both sensors were characterised with a calibrated transducer. The scanning area on each sensor was $12 \times 16.2 \mathrm{~mm}$, at a step size of $100 \mu \mathrm{m}$ and a temporal resolution of 8 ns. A fibre-coupled, Nd:YAG pumped, wavelength-tunable optical parametric oscillator (OPO) system (GWU, Spectra-Physics, Santa Clara, CA, USA) was used for delivery of excitation light, providing $7 \mathrm{~ns}$ pulses at $10 \mathrm{~Hz}$ pulse repetition rate and a pulse energy of $\sim 19 \mathrm{~mJ}$ at $800 \mathrm{~nm}$ (at the distal end of the fibre). The excitation source was positioned above the phantom as shown in Figure 2. To characterise the beam profile, an acetate sheet with a grid of dots was placed on the medium surface and photoacoustically imaged at $800 \mathrm{~nm}$. The 1/e radius of the approximately Gaussian beam profile was $\sim 4 \mathrm{~mm}$. Data was acquired for 9 wavelengths: 750, 780, $810,860,910,960,1010,1060$ and $1110 \mathrm{~nm}$. The pulse energy at the output of the laser was measured from pulse-to-pulse using an integrating sphere, and related to powermeter measurements of pulse energy at the distal end of the fibre (in Joule).

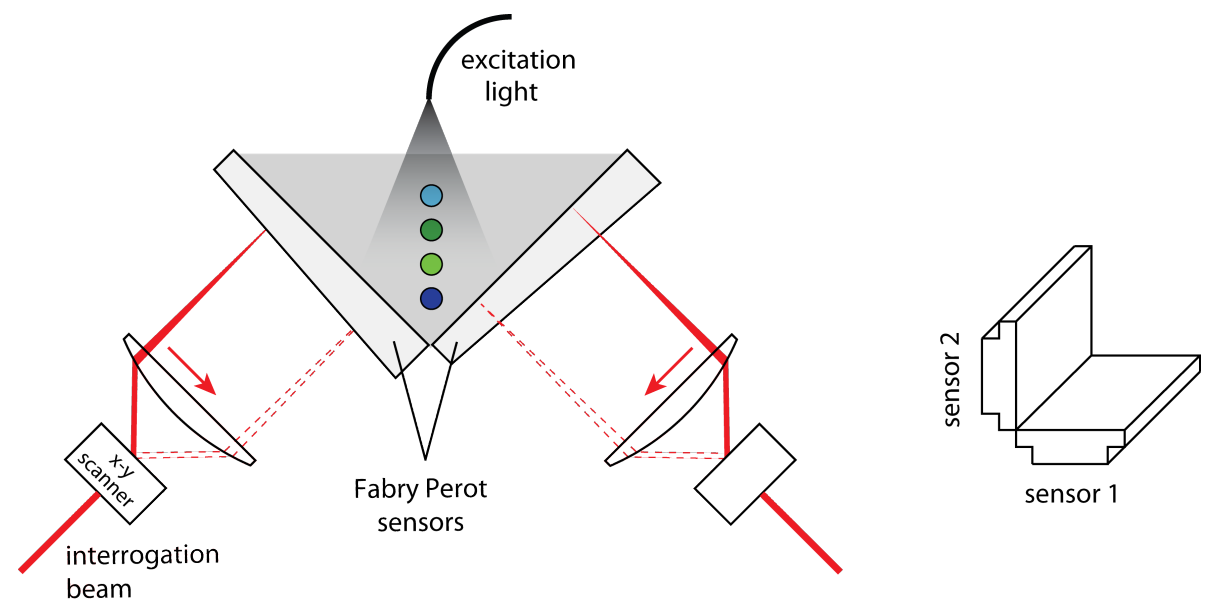

Figure 2: Left - Schematic of experimental setup, in cross-section. Right - orthogonal arrangement of sensors. 


\section{ACOUSTIC RECONSTRUCTION}

The measured time-series were pre-processed prior to image reconstruction. First, a baseline correction was employed to all time-series by subtracting from each the respective median value of time-samples between $392-$ 1192 ns. The time-series were then low-pass filtered up to $15 \mathrm{MHz}$. Finally, the noisiest channels were eliminated - this was defined as 5\% of the channels with the highest variance in baseline (evaluated again between 392$1192 \mathrm{~ns})$. An iterative time-reversal image reconstruction scheme, including the dual constraints of non-negative initial pressure and zero initial particle velocity, was used to obtain the images of the initial acoustic pressure in Pascal. ${ }^{15,16}$ A pseudospectral time-domain propagation model, $\mathrm{k}$-Wave ${ }^{17}$ was used for both the time-reversal and as the forward model. The algorithm was run for 20 iterations to obtain a $13.2 \times 17.9 \times 18 \mathrm{~mm}^{3}$ volumetric image of initial pressure with $50 \mu \mathrm{m}$ voxel size, for each of the 9 wavelengths. Figure 3 shows the resulting initial pressure map for the $1060 \mathrm{~nm}$ acquisition. The tube structures are successfully recovered with minimal limited view artefacts - although some artefacts due to reflections from the tubes are visible.

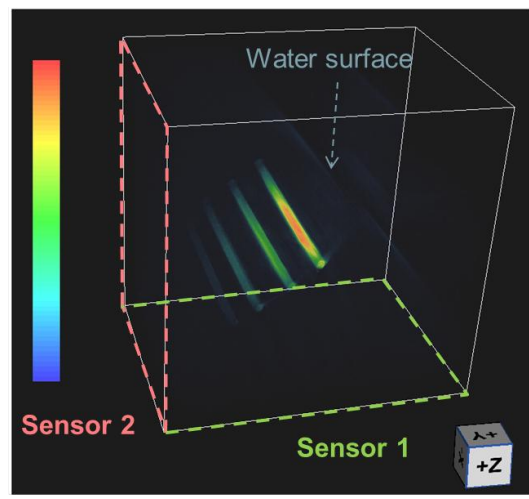

(a)
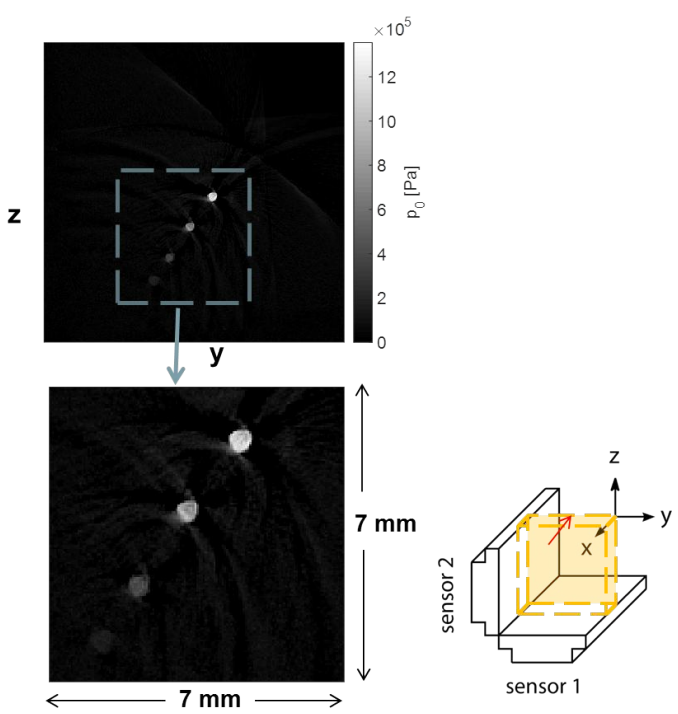

(b)

Figure 3: Acoustic reconstruction at $1060 \mathrm{~nm}$. (a) Volume rendering of the $13.2 \times 17.9 \times 18 \mathrm{~mm}^{3}$ volume. Pressure amplitude is colour encoded. Opacity increases linearly. (b) Maximum-intensity-projection of the central $1 \mathrm{~mm}$ YoZ cross-section, and $7 \times 7 \mathrm{~mm}$ inset of the tube region. Colour-bar represents pressure in Pascal, for the range 0-1.43 $\mathrm{MPa}$.

\section{OPTICAL RECONSTRUCTION}

In order to recover chromophore concentrations from the measured multiwavelength initial pressure maps, a model-based inversion framework was employed. This optical reconstruction was performed for a cuboid region of interest (region-of-interest (ROI)), $10 \mathrm{~mm}$ deep from the surface of the medium, and with lateral dimensions of $8 \times 8 \mathrm{~mm}$ aligned with the centre of the illumination beam at the surface. Two meshes, coarse and fine, were created from tetrahedral elements with respectively $200 \mu \mathrm{m}$ and $100 \mu \mathrm{m}$ average element size, and a total of 60247 and 397441 nodes respectively. The error functional to be minimised was defined as:

$$
\varepsilon=\sum_{l=1}^{L} \sum_{h=1}^{N h}\left[p_{0, h}^{\text {meas }}\left(\lambda_{l}\right)-p_{0, h}^{\text {model }}\left(\mathbf{c}_{k}, \lambda_{l}\right)\right]^{2}
$$

where subscripts $l$ and $h$ denote wavelength index and nodal position index respectively, ${ }^{18} \mathbf{c}_{k}$ denotes the unknown concentration distributions for a set of $k$ chromophores, and $p_{0, h}^{\text {meas }}\left(\lambda_{l}\right)$ is the experimental initial pressure distribution interpolated from the k-Wave grid (acoustic model) into the nodes of the mesh (optical model). $p_{0, h}^{\text {model }}\left(\mathbf{c}_{k}, \lambda_{l}\right)$ is the modelled initial pressure distribution given by

$$
p_{0}^{\text {model }}\left(\mathbf{c}_{k}, \lambda_{l}\right)=\Gamma\left(\mathbf{c}_{k}\right) \Phi\left(\mathbf{c}_{\mathbf{k}}, \mu_{s}^{\prime}\right) \mu_{a}\left(\mathbf{c}_{k}\right),
$$


where

- $\Gamma\left(\mathbf{c}_{k}\right)$ is the Grüneisen parameter defined as a linear combination of the chromophore concentrations, modelled using Equation 2;

- $\mu_{a}\left(\mathbf{c}_{k}\right)=\sum_{i=1}^{k} \alpha_{i} \mathbf{c}_{i}$ is the optical absorption with known chromophore spectra $\alpha(\lambda)$ obtained as described in Section 2;

- $\Phi\left(\mathbf{u}, \mu_{s}^{\prime}\right)$ is the light fluence computed using the 3D diffusion approximation to the radiative transfer equation (RTE) (Toast $++{ }^{19}$ ), with reduced scattering coefficient and beam location fixed and known through experimental characterisation.

The unknowns to be estimated were the spatial distributions $\mathbf{c}_{k}$ of the four chromophores of interest - water, india ink, $\mathrm{CuSO}_{4}$ and $\mathrm{NiSO}_{4}$. The total number of unknowns was therefore 1589764 for the $100 \mu \mathrm{m}$ mesh implementation and 240988 for the $200 \mu \mathrm{m}$ mesh implementation. The minimisation algorithm was the limitedmemory BFGS (L-BFGS), ${ }^{20}$ and the functional gradients, $\partial \varepsilon / \partial c_{k}$, were calculated with the adjoint method. ${ }^{21,22}$

\section{RESULTS}

The unknowns were initialised to the background values and the minimisation was allowed to run for 400 iterations.
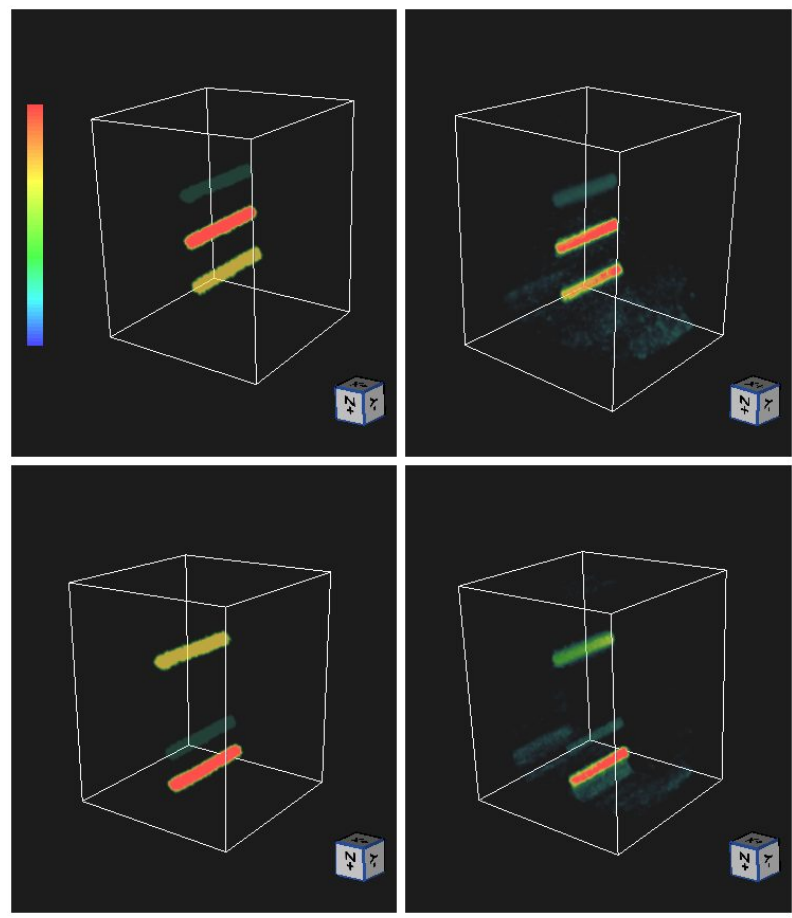

(a)
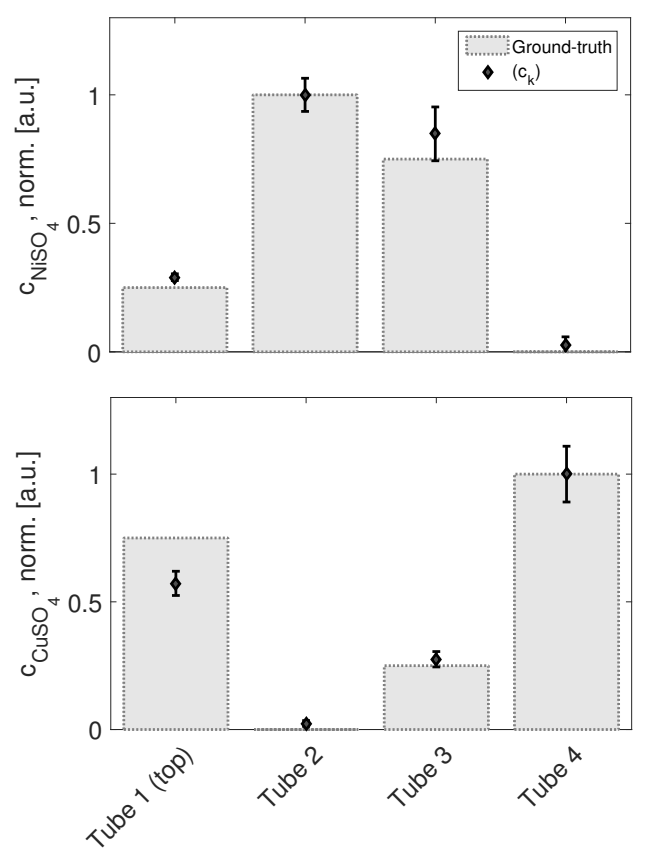

(b)

Figure 4: Comparison between true and $\left(\mathbf{c}_{\mathbf{k}}\right)$ model-based inversion estimated normalised concentration mappings of $\mathrm{NiSO}_{4}$ and $\mathrm{CuSO}_{4}$, displayed as (a) volume rendering; (b) tube-by-tube metric . Colour-bar ranges between zero and one, where in each case one is matched to the mean intensity of the tube with highest concentration

Volume renderings of the chromophore distribution estimates are shown in Figure 4(a), normalised to the mean intensity of the tube with highest concentration, showing the degree of agreement between the groundtruth and the estimates in terms of relative concentration. Figure 4(b) plots the mean and standard deviation of the normalised concentrations - labelled $\left(\mathbf{c}_{\mathbf{k}}\right)$ - for each tube (a cylindrical region of length $4 \mathrm{~mm}$ and diameter 
$150 \mu \mathrm{m}$ within each tube was used to avoid, respectively, model discrepancies expected close to the physical boundary of the ROI and mesh interpolation errors). The estimation procedure was able to correctly detect the presence of $\mathrm{NiSO}_{4}$ in the top three tubes, and also found low levels of $\mathrm{NiSO}_{4}$ in the bottom tube (low cross-talk/ false-positives). Furthermore, there is good agreement in the relative concentrations, with the main deviations occurring for the third tube, over-estimated on average by an absolute error of $9.8 \%$. Similar trends are seen for $\mathrm{CuSO}_{4}$, in terms of accurate detection, reduced cross-talk and relative inter-tube agreement. In this case, the main deviation was seen for the first tube, under-estimated on average by an absolute error of $17.8 \%$.

Figure 5 shows the estimates that were obtained for the ratio of absolute chromophore concentrations, $Q$ (Equation 1), a quantity analogous to $\mathrm{SO}_{2}$. Estimates for the four tubes were $31.8 \pm 1.6,97.5 \pm 1.1,73.7 \pm 2.7$ and $2.5 \pm 2.8$ (mean \pm standard deviation of the point cloud), for ground-truth values of $25,100,75$ and $0 \%$ respectively.

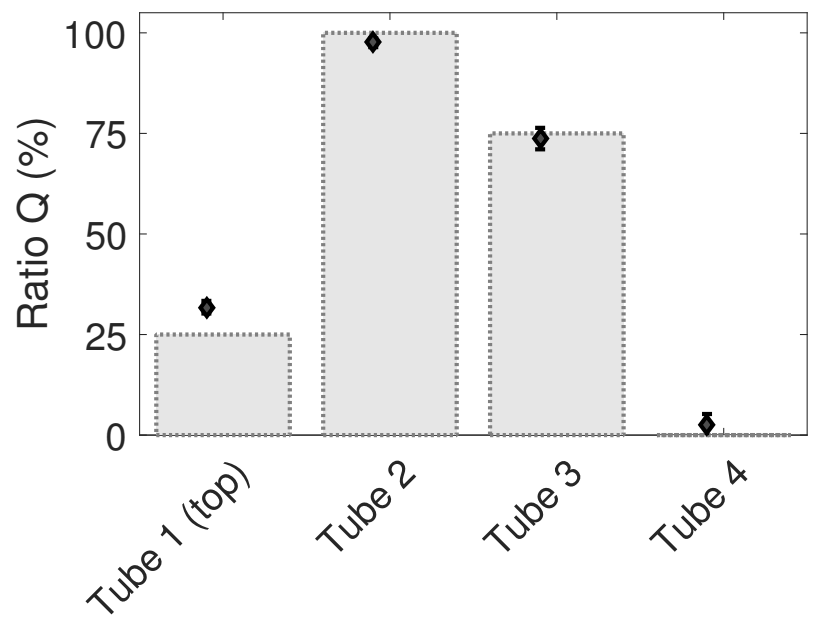

Figure 5: Ground-truth for ratio $\mathrm{Q}(\%)$ is compared to $\left(\mathbf{c}_{\mathbf{k}}\right)$-inversion outcomes.

Despite the promising outcomes for the normalised concentration estimates, and the estimates of the chromophore concentration ratios, the estimates in absolute units (concentrations in Molar) do not agree as well, with an overall overestimation in all cases - see Figure $6,\left(\mathbf{c}_{\mathbf{k}}\right)$ vs ground-truth. As one potentially significant source of uncertainty is the set of operations that relate the raw measured time-series to meaningful units of pressure $(\mathrm{Pa})$ per incident light fluence (in $\mathrm{Jm}^{-2}$ ), a new inversion procedure was devised, inverting for the previous unknowns but also for an additional calibration parameter $K$ (a single scalar), ${ }^{3,23}$ acting as a global scaling of the modeled $p_{0}$ in equation 4 . This strategy, here named $\left(\mathbf{c}_{\mathbf{k}}, K\right)$-inversion, was run using the initialisation values of the outcomes of the previous inversion strategy. Due to the slow rate of convergence, these results were run on the $200 \mu \mathrm{m}$ mesh and only stopped when the infinity norm of the gradient reached machine-precision. Figure 6 shows an improvement in estimates of absolute concentration with the $\left(\mathbf{c}_{\mathbf{k}}, K\right)$-inversion, compared to the $\left(\mathbf{c}_{\mathbf{k}}\right)$-inversion only. New estimates are promising especially for $\mathrm{CuSO}_{4}$ content, with main deviations seen only for the $3^{\text {rd }}$ tube. 

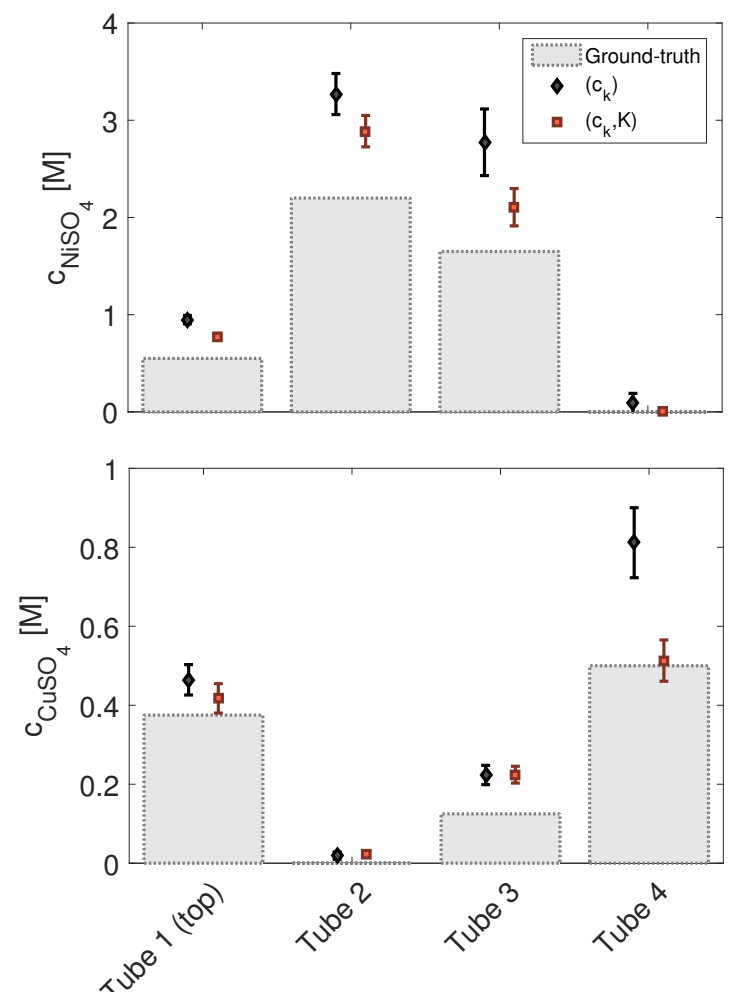

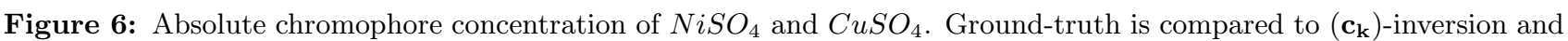
$\left(\mathbf{c}_{\mathbf{k}}, K\right)$-inversion

\section{EFFECT OF UNCERTAINTIES ON QUANTIFICATION}

The success of a quantification framework depends on dealing with each of the stages of the inversion as accurately as possible (system calibration, acoustic reconstruction, thermoelastic conversion and optical reconstruction) and having access to accurate parameter estimates. This section briefly looks at the size of the errors that would have occurred if individual aspects related to system calibration, acoustic reconstruction, thermoelastic efficiency and light model input parameters had not been explicitly optimised. Table 1 shows comparison for an error metric, $\delta_{\text {tubes }}=\frac{\left\|c_{\text {true }}^{(\text {norm })}-c_{\text {est }}^{(\text {norm })}\right\|}{\left\|c_{\text {true }}^{(\text {norm })}\right\|} \times 100(\%)$, where $c_{\text {true }}^{(\text {norm })}$ is the true normalised concentration and $c_{\text {est }}^{(\text {norm })}$ the estimated normalised concentrations after 400 iterations of the $\left(\mathbf{c}_{\mathbf{k}}\right)$ inversion. As before, only values within the selection mask are considered and normalisation is done to the average of the brightest tube. This is not intended as a rigorous sensitivity study, but rather serves to illustrate that it is necessary to take care to obtain best estimates of the various model parameters as they can have a significant deleterious effect on the concentration estimates.

The default scenario (i.e. the scenario where no aspects or parameters were perturbed) yielded, according to the chosen metric, a $6.5 \%$ error for $\mathrm{NiSO}_{4}$ and a $14.7 \%$ error for $\mathrm{CuSO}_{4}$. All alterations towards nonoptimised scenarios led to an increase in this error. When the Grüneisen was not correctly accounted for but rather set fixed to background $\Gamma=\Gamma_{\mathrm{H}_{2} \mathrm{O}}$, this led to a $39.6 \%$ error versus $6.5 \%$ in the default situation. Such an outcome is not unexpected, but worth highlighting given that many PAT quantification strategies assume $\Gamma$ known and constant when in fact it varies considerably among tissue types and even within a given tissue (e.g. in blood it has been shown to follow a linear relation with hemoglobin concentration). ${ }^{24}$ When no calibration was available, i.e. $p_{0}$ could not be represented in Pascal but remained in arbitrary units, the situation was handled by normalising the maximum of the experimental $p_{0}$ data to the maximum of the $p_{0, \text { model }}\left(\mathbf{c}_{k, \text { true }}\right)$, where $\mathbf{c}_{k, \text { true }}$ were the ground-truth estimates measured independently. In this experiment such an approach was not successful - whether because of noise, model mismatch, compounded effect of other uncertainties - and 


\begin{tabular}{ll} 
Source of explicit uncertainty/error & $\delta_{\text {tubes }, \mathrm{NiSO}_{4}}$ \\
\hline None & $6.5 \%$ \\
\hline$\mu_{s}^{\prime}: 20 \%$ overestimation & $7.4 \%$ \\
\hline Grüneisen: $\Gamma=\Gamma_{\mathrm{H}_{2} \mathrm{O}}$ & $39.6 \%$ \\
\hline No acoustic pressure calibration & $14.4 \%$ \\
\hline $\begin{array}{l}\text { One-step (non-iterative) time reversal } \\
\text { One-step (non-iterative) }\end{array}$ & $26.5 \%$ \\
\hline sensor 1 only & $26.4 \%$ \\
\hline
\end{tabular}

Table 1: Error in normalised $\mathrm{NiSO}_{4}$ concentration is compared between the default case and various scenarios where a given parameter or aspect is perturbed.

therefore, since the model-based inversion had not been designed to account for calibration deviations, led to errors in concentration estimates of $14.4 \%$. Though this strategy could in principle be successful in phantom experiments, it requires the use of prior knowledge of $\mathbf{c}_{k, t r u e}$, which will be the unknown parameter in an in vivo setting. Finally, 26.5\% error in the concentration metric was obtained when using non-iterative time-reversal image reconstruction. The majority of this error is due to the ability of iterative time-reversal to correct for the partial detection surface, and because its positivity constraint removes negative artefacts. Another limited-view related case was computed, where it was considered data had only been acquired from only one planar array, and this led to a similar level of error.

\section{CONCLUSIONS}

The aim of the work was to obtain highly-resolved, volumetric mappings of chromophore distribution from experimental PAT data, through the use of a model-based inversion scheme. Promising estimates of normalised tube concentration were attained. For the ratiometric quantity $Q(\%)$, tube error did not exceed 8.5 percentage points (considering datapoints up to one standard deviation of the mean of each tube estimate). In terms of absolute quantification, it was shown that including a scalar scaling parameter in the inversion showed promise in improving estimates to some extent, although there is still room for further improvement. Further analysis also showed that, as important as it is to include a model of light transport that deals with the presence of spectral colouring, it is equally important to deal adequately with the earlier stages of the inverse problem: e.g. to have an accurate map of initial pressure distribution, to correctly account for thermoelastic efficiency, and to have a well calibrated measurement system.

\section{ACKNOWLEDGMENTS}

The work presented was supported by a CASE studentship jointly funded by the UK's Engineering and Physical Sciences Research Council and National Physical Laboratory.

\section{REFERENCES}

[1] Weber, J., Beard, P. C., and Bohndiek, S. E., "Contrast agents for molecular photoacoustic imaging," Nature Methods 13(8), 639-650 (2016).

[2] Laufer, J., Delpy, D., Elwell, C., and Beard, P., "Quantitative spatially resolved measurement of tissue chromophore concentrations using photoacoustic spectroscopy : application to the measurement of blood oxygenation and haemoglobin concentration," Phys Med Biol 52(1), 141-168 (2007).

[3] Laufer, J., Cox, B., Zhang, E., and Beard, P., "Quantitative determination of chromophore concentrations from 2D photoacoustic images using a nonlinear model-based inversion scheme.," Applied optics 49(8), 1219-33 (2010).

[4] Brochu, F. M., Brunker, J., Joseph, J., Tomaszewski, M. R., Morscher, S., and Bohndiek, S. E., "Towards Quantitative Evaluation of Tissue Absorption Coefficients Using Light Fluence Correction in Optoacoustic Tomography," IEEE transactions on medical imaging 36(1), 322-331 (2017). 
[5] Palmer, K. F. and Williams, D., "Optical properties of water in the near infrared," Joural Optical Society of America 64(8), 1107-1110 (1974).

[6] Prahl, S. A., van Gemert, M. J. C., and Welch, A. J., "Determining the optical properties of turbid media by using the adding-doubling method," Applied Optics 32(4), 559 (1993).

[7] Stahl, T., Allen, T., and Beard, P., "Characterization of the thermalisation efficiency and photostability of photoacoustic contrast agents," Proc of SPIE 8943, 89435H-1 - 89435H-8 (2014).

[8] Bilaniuk, N. and Wong, G. S. K., "Speed of sound in pure water as a function of temperature," The Journal of the Acoustical Society of America 93(3), 1609-1612 (1993).

[9] Laufer, J., Zhang, E., and Beard, P., "Evaluation of Absorbing Chromophores Used in Tissue Phantoms for Quantitative Photoacoustic Spectroscopy and Imaging," IEEE Journal of Selected Topics in Quantum Electronics 16(3), 600-607 (2010).

[10] Zhang, E., Laufer, J., and Beard, P., "Backward-mode multiwavelength photoacoustic scanner using a planar Fabry-Perot polymer film ultrasound sensor for high-resolution three-dimensional imaging of biological tissues.," Applied optics 47(4), 561-577 (2008).

[11] Ellwood, R., Ogunlade, O., Zhang, E. Z., Beard, P. C., and Cox, B. T., "Orthogonal Fabry-Pérot sensors for photoacoustic tomography," in [Proceedings of SPIE], Oraevsky, A. A. and Wang, L. V., eds., 9708, 97082N (2016).

[12] Ellwood, R., Ogunlade, O., Zhang, E., Beard, P., and Cox, B., "Photoacoustic tomography using orthogonal Fabry Pérot sensors," Journal of Biomedical Optics 22(4), 041009 (2017).

[13] Paltauf, G., Nuster, R., Haltmeier, M., and Burgholzer, P., "Experimental evaluation of reconstruction algorithms for limited view photoacoustic tomography with line detectors," Inverse Problems 23(6), S81S94 (2007).

[14] Xu, Y., Wang, L. V., Ambartsoumian, G., and Kuchment, P., "Reconstructions in limited-view thermoacoustic tomography," Medical Physics 31(4), 724 (2004).

[15] Stefanov, P. and Uhlmann, G., "Thermoacoustic tomography with variable sound speed," Inverse Problems 25(7), 075011 (2009).

[16] Paltauf, G., Viator, J. a., Prahl, S. a., and Jacques, S. L., "Iterative reconstruction algorithm for optoacoustic imaging," The Journal of the Acoustical Society of America 112(4), 1536 (2002).

[17] Treeby, B. E. and Cox, B. T., "k-Wave: MATLAB toolbox for the simulation and reconstruction of photoacoustic wave fields," Journal of Biomedical Optics 15(2), 021314 (2010).

[18] Malone, E., Cox, B. T., Arridge, S., Malone, E., Powell, S., Cox, B. T., and Arridge, S., "Reconstructionclassification method for quantitative photoacoustic tomography," Journal of Biomedical Opics 20(12), 126004-1 (2015).

[19] Schweiger, M. and Arridge, S., "The Toast++ software suite for forward and inverse modeling in optical tomography.," Journal of Biomedical Optics 19(4), 040801 (2014).

[20] Nocedal, J. and Wright, S. J., [Numerical Optimization], Springer, New York, 2nd ed. (2006).

[21] Cox, B. T., Arridge, S. R., and Beard, P. C., "Gradient-based quantitative photoacoustic image reconstruction for molecular imaging," Proceedings of SPIE 6437, 64371T-64371T-10 (2007).

[22] Soonthornsaratoon, T., Gradient-based methods for quantitative photoacoustic tomography, PhD thesis, University College London, London, UK (2014).

[23] Fonseca, M., Saratoon, T., Zeqiri, B., Beard, P., and Cox, B., "Sensitivity of quantitative photoacoustic tomography inversion schemes to experimental uncertainty," in [Proceedings of SPIE], Oraevsky, A. A. and Wang, L. V., eds., 9708(0), 97084X (2016).

[24] Yao, D.-k., Zhang, C., Maslov, K., and Wang, L. V., "Photoacoustic measurement of the Grüneisen parameter of tissue.," Journal of Biomedical Optics 19(1), 017007 (2014). 